\title{
DUAL SPACES OF WEIGHTED SPACES
}

\author{
BY \\ W. H. SUMMERS
}

\begin{abstract}
The topological duals of a large class of weighted spaces of continuous functions are characterized as spaces of Radon measures which can be factored into a product of a weight function and a bounded Radon measure. We next obtain a representation for a base for the equicontinuous subsets of these dual spaces and for the extremal points of the members of this base. Finally, among other applications, these ideas make possible an extension of the representation theorem for biequicontinuous completed tensor products of weighted spaces obtained by the author in an earlier paper.
\end{abstract}

1. Introduction. The role of weighted spaces of continuous functions in approximation theory is well known (e.g., see Nachbin [7], [8]). Moreover, weighted spaces as locally convex spaces have proved to be a useful tool in the study of entire functions (see Taylor [14]) and other mathematical objects. For example, Buck [1], Collins and Dorroh [2], and Conway [3], [4], among others, have implemented the notion that the weighted space $\left(C_{b}(X), \beta\right)$ of bounded continuous functions on a locally compact Hausdorff space $X$ with the strict topology $\beta$ is the proper setting to study the space $C_{b}(X)$ as a locally convex space if one wishes to relate its properties with those of $X$. One of the key results in this direction was the identification by Buck [1] of the topological dual $\left(C_{b}(X), \beta\right)^{*}$ with the space of bounded Radon measures on $X$. We should also mention that a recent representation theorem due to Prolla [9] manifests the universal nature of weighted spaces in the class of all locally convex spaces.

It is the purpose of this paper to continue the investigation of weighted spaces as locally convex spaces that was initiated in [13]. In particular, it is shown that our characterization of the topological duals of certain weighted spaces as spaces of Radon measures in [13] can be extended to a larger class of weighted spaces (we are now able to remove a continuity condition imposed in [13]), and several examples are given to help delineate the situation. Moreover, we give characterizations analogous to those given in [13] for a base for the equicontinuous sets in the topological duals of this larger class of weighted spaces and the extremal points of the members of this base. Finally, various applications of the preceding results

Presented to the Society, August 27, 1969; received by the editors September 29, 1969.

AMS 1969 Subject Classifications. Primary 4625, 4601; Secondary 2830.

Key Words and Phrases. Weighted spaces of continuous functions, strict topology, Radon measure, equicontinuous sets, extremal points, Nachbin family, factorization, biequicontinuous completed tensor product, Mackey space. 
are made; these include a generalization of our representation theorem for the biequicontinuous completed tensor product of weighted spaces given in [13].

The contents of this paper are, for the most part, either results or generalizations of results from the author's Ph.D. dissertation written under the direction of Professor Heron S. Collins at Louisiana State University (1968). The author wishes to express his gratitude to Professor Collins for his advice and encouragement.

2. Preliminaries. The underlying topological spaces $(X$ and $Y$ ) in the sequel will be at least completely regular $T_{1}$-spaces, while all functions will be complexvalued unless explicitly stated otherwise. We shall let $C(X)$ denote the space of all continuous functions on $X, B(X)$ denote the space of all bounded functions on $X$, and $B_{0}(X)$ denote the space of all functions on $X$ which vanish at infinity. Further, let $C_{b}(X)=C(X) \cap B(X)$ and $C_{0}(X)=C(X) \cap B_{0}(X)$, while $C_{c}(X)$ will denote the space of all continuous functions on $X$ which have compact support. If $f$ is a function on $X$, then $N(f)=\{x \in X: f(x) \neq 0\}$ and the closure of $N(f)$ is denoted spt $(f)$. Moreover, if $S \subseteq X$, then $R(f ; S)$ will denote the restriction of $f$ to $S$, and if $R(f ; S) \in B(S)$, then define $\|f\|_{s}=\sup \{|f(x)|: x \in S\}$. In the case $S=X$ we write $\|f\|$, and will refer to the topology induced by $\|\cdot\|$ on $B(X)$ as the uniform topology.

A Nachbin family $V$ on $X$ is a set of nonnegative upper semicontinuous (u.s.c.) functions on $X$ satisfying the condition that if $u, v \in V$ and $\lambda \geqq 0$, then there is a $w \in V$ such that $\lambda u, \lambda v \leqq w$ (pointwise). If $V$ is a Nachbin family on $X$, then the corresponding weighted space

$$
C V_{0}(X)=\left\{f \in C(X): f v \in B_{0}(X) \text { for every } v \in V\right\}
$$

endowed with the weighted topology $\omega$ (or $\omega_{V}$ ) generated by the seminorms $\left\{p_{v}: v \in V\right\}$ where $p_{v}(f)=\|f v\|$ for each $f \in C V_{0}(X)$. Now $C V_{0}(X)$ is a locally convex (topological vector) space with a base of closed and absolutely convex neighborhoods of zero formed by the sets $V_{v}=\left\{f \in C V_{0}(X):\|f v\| \leqq 1\right\}$ for each $v \in V$. If $U$ and $V$ are two Nachbin families on $X$ and if for each $u \in U$ there is a $v \in V$ such that $u \leqq v$, then we write $U \leqq V$. In case $U \leqq V$ and $V \leqq U$, we write $U \approx V$.

Unless otherwise specified, we will consider only Nachbin families $V$ on $X$ for which $C V_{0}(X)$ is Hausdorff (necessary and sufficient conditions for this, as well as examples of weighted spaces are given in [13]). We should note at this point that requiring $C V_{0}(X)$ to be Hausdorff can yield information about $X$ in certain cases. In fact, requiring $C V_{0}(X)$ to be Hausdorff can be very close to requiring that $X$ be locally compact.

2.1. THeOREM. If $V$ is a Nachbin family on $X$ with $C_{c}^{+}(X) \leqq V \subseteq C_{0}^{+}(X)$, then $C V_{0}(X)$ is Hausdorff if and only if $X$ contains a dense locally compact (and hence open) subspace $Y$. 
Proof. Assume $Y$ is a dense locally compact subspace of $X$; thus $Y$ is open in $X$ [6, p. 45]. If $f \in C V_{0}(X)$ with $f \neq 0$, then there is an $x \in Y$ with $f(x) \neq 0$, and hence there is an open neighborhood $A$ of $x$ with $A \subseteq Y$ and such that cl $(A)$ is compact. Consequently, there exists a $\phi \in C_{c}^{+}(X)$ with $\phi(x)>0$, and this implies there is a $v \in V$ such that $\phi \leqq v$ from which it follows that $|f(x)| v(x)>0$.

Now assume $C V_{0}(X)$ is Hausdorff. If $y \in X$ and if $A$ is an open neighborhood of $y$, then there exists a $\phi \in C(X)$ such that $0 \leqq \phi \leqq 1, \phi(y)=1$, and $\phi(X \backslash A)=0$. If $v \in V$ and $\varepsilon>0$, then $K=\{x \in X: \phi(x) v(x) \geqq \varepsilon\} \subseteq\{x \in X: v(x) \geqq \varepsilon\}$, and this implies $K$ is compact. Hence $\phi \in C V_{0}(X)$, and since $\phi \neq 0$ there is a $v \in V$ with $\phi v \neq 0$; i.e., $N(v) \cap A$ is not void. Therefore $Y=\bigcup\{N(v): v \in V\}$ is dense in $X$; and if $y \in Y$, then there exists $v \in V$ such that $y \in B=\{x \in X: v(x)>(1 / 2) v(y)\}$. But $B$ is open, $\mathrm{cl}(B)$ is compact, and $\mathrm{cl}(B) \subseteq Y$ which completes the proof.

For example, if we let $X$ denote the rationals with the topology induced by the usual topology on the reals, then $X$ is a completely regular $T_{1}$-space which does not have a dense locally compact subspace. In particular, if $V$ is a Nachbin family on $X$ with $C_{c}^{+}(X) \leqq V \subseteq C_{0}^{+}(X)$, then $V=\{0\}$ and $C V_{0}(X)$ is $C(X)$ with the indiscrete topology.

3. The dual space of $C V_{0}(X)$. In [13], the author characterized the space $C V_{0}(X)^{*}$ in the case $V$ was a Nachbin family on a locally compact space $X$ which satisfied the condition that $V \subseteq C^{+}(X)$. We are now able to generalize this result to a much larger class of Nachbin families on locally compact spaces (Theorem 3.1).

We will denote the complex Radon measures on a locally compact space $X$ by $M(X)$, while $M_{b}(X)$ will denote the subspace of bounded Radon measures. Now if $V$ is a Nachbin family on $X$, then each $v \in V$ is Borel measurable and bounded on the compact subsets of $X$. Define $V \cdot M_{b}(X)$ to be $\{v \cdot \mu: v \in V$, $\left.\mu \in M_{b}(X)\right\}$ where $v \cdot \mu$ is the Radon measure on $X$ defined by $v \cdot \mu(f)=\mu(f v)$ for $f \in C_{c}(X)$. Therefore $V \cdot M_{b}(X)$ is always contained in $M(X)$, and will actually be a linear subspace of $M(X)$ for a large class of Nachbin families $V$ on $X$, although this is by no means obvious from the definition. In fact, $V \cdot M_{b}(X)$ is our candidate for $C V_{0}(X)^{*}$ in the case of the Nachbin families to be considered, but, of course, we do not expect uniqueness of representation (see Example 3.6).

3.1. THEOREM. If $X$ is locally compact and if $V$ is a Nachbin family on $X$ with $V \leqq C^{+}(X)$, then $V \cdot M_{b}(X)$ is a linear subspace of $M(X)$ and $T: V \cdot M_{b}(X) \rightarrow C V_{0}(X)^{*}$ is a (linear) onto isomorphism where $T(v \cdot \mu)(f)=\int f v d \mu$ for each $f \in C V_{0}(X)$. In this case we write $C V_{0}(X)^{*}=V \cdot M_{b}(X)$.

The proof of Theorem 3.1 will be deferred until after the following lemmas have been established.

3.2. LEMMA. If $X$ is locally compact and if $V=C^{+}(X)$, then $C_{c}(X) \subseteq C V_{0}(X)$ $\subseteq C_{b}(X), \omega_{V}$ is finer than the uniform topology on $C V_{0}(X)$, and the inductive limit topology on $C_{c}(X)$ is finer than the topology induced on $C_{c}(X)$ by $\omega_{V}$. 
Proof. $C_{c}(X)$ is always contained in $C V_{0}(X)$, while it is clear in this case that $C V_{0}(X) \subseteq C_{b}(X)$. Moreover, it is immediate from [13, Theorem 3.1] that $\omega_{V}$ is finer than the uniform topology. Now let $v \in V$, and for every compact $K$ in $X$ let $B_{K}=\left\{f \in C_{c}(X): \operatorname{spt}(f) \subseteq K,\|f\| \leqq\left(\|v\|_{K}\right)^{-1}\right\}$ if $N(v) \cap K$ is nonvoid and let $B_{K}=\left\{f \in C_{c}(X): \operatorname{spt}(f) \subseteq K\right\}$ if $N(v) \cap K$ is void. Since the absolutely convex hull $A$ of $\bigcup\left\{B_{K}: K\right.$ compact $\}$ is an inductive limit neighborhood of zero in $C_{c}(X)$ [10, p. 79], it will suffice to show that $f \in A$ implies $f \in V_{v} \cap C_{c}(X)$. If $f \in A$, then there exist scalars $\left\{\lambda_{k}\right\}_{k=1}^{n}$ and functions $\left\{f_{k}\right\}_{k=1}^{n}$ with $\sum_{k=1}^{n}\left|\lambda_{k}\right| \leqq 1$, and $f_{k} \in B_{K_{k}}, k=1, \ldots, n$, such that $f=\sum_{k=1}^{n} \lambda_{k} f_{k}$. Hence

$$
|f(x)| v(x) \leqq \sum_{k=1}^{n}\left|\lambda_{k}\right| \cdot\left|f_{k}(x)\right| v(x) \leqq \sum_{k=1}^{n}\left|\lambda_{k}\right| \leqq 1,
$$

and this implies $\|f v\| \leqq 1$.

3.3. Lemma. Let $X$ be locally compact and let $v$ be a nonnegative u.s.c. function on $X$. If $\nu \in M^{+}(X)$ has the property that $\int f d \nu \leqq 1$ for every $f \in C_{c}^{+}(X)$ with $\|f v\| \leqq 1$, then there exists $\mu \in M_{b}^{+}(X)$ with $\|\mu\| \leqq 1$ and such that $\nu=v \cdot \mu$.

Proof. Define $u$ on $X$ by

$$
\begin{aligned}
u(x) & =(1 / v)(x), & & x \in N(v), \\
& =+\infty, & & x \in X \backslash N(v),
\end{aligned}
$$

and note that $u$ is a nonnegative lower semicontinuous function on $X$. If $\phi \in C_{c}^{+}(X)$ and if $\phi \leqq u$, then $\|\phi v\| \leqq 1$ which implies $\int \phi d \nu \leqq 1$. Consequently,

$$
\sup \left\{\int \phi d v: \phi \in C_{c}^{+}(X), \phi \leqq u\right\} \leqq 1,
$$

and this implies the upper integral of $u$ with respect to $v$ is finite. Hence $u$ is $v$ integrable from which it follows that $\nu(X \mid N(v))=0$ and $\int u d \nu=\int(1 / v) d \nu \leqq 1$; i.e., the measure $u \cdot \nu=(1 / v) \cdot \nu$ is the desired $\mu$.

Proof of 3.1. Since $C_{c}(X)$ is an $\omega_{V}$-dense subspace of $C V_{0}(X)$ [8, p. 64], it follows that $T$ is a well-defined one-to-one function into $C V_{0}(X)^{*}$. We next show that $T$ is onto, and to this end, fix $L \in C V_{0}(X)^{*}$. Now the inductive limit topology on $C_{c}(X)$ is finer than the topology induced on $C_{c}(X)$ by $\omega_{V}$ from Lemma 3.2 and [13, Theorem 3.1], and so $R\left(L ; C_{c}(X)\right) \in\left(C_{c}(X)\right.$, ind lim)*. Therefore there exists a unique $\mu \in M(X)$ such that $R\left(L ; C_{c}(X)\right)(f)=\int f d \mu$ for $f \in C_{c}(X)$. Write $\mu$ uniquely as $\alpha+i \beta$ where $\alpha$ and $\beta$ are real Radon measures on $X$, and let $\alpha=\alpha^{+}-\alpha^{-}$ be the minimal decomposition of $\alpha$. Since $L \in C V_{0}(X)^{*}$, there is a $v \in V$ such that $|L(f)| \leqq 1$ for every $f \in V_{v}$; thus if $f \in C_{c}(X) \cap V_{v}$, then $\int f d \alpha \leqq\left|\int f d \mu\right| \leqq 1$ from whence $\int f d \alpha^{+} \leqq 1$. By Lemma 3.3 there is a $\sigma \in M_{b}(X)$ such that $\alpha^{+}=v \cdot \sigma$ (with $\|\sigma\| \leqq 1)$, and it easily follows that there is a $\tau \in M_{b}(X)$ such that $\alpha^{-}=v \cdot \tau$. It is now clear that there exists a $\nu \in M_{b}(X)$ such that $\mu=v \cdot v$ (we will show in $\$ 4$ that $\nu$ can be chosen so that $\|\nu\| \leqq 1)$. Hence $\mu \in V \cdot M_{b}(X)$, and since $T(\mu)$ agrees with $L$ 
on an $\omega_{V}$-dense subspace of $C V_{0}(X)$ we have that $T(\mu)=L$. It remains to show $V \cdot M_{b}(X)$ is a linear subspace of $M(X)$ and that $T$ is linear. To see this, first note that $V \cdot M_{b}(X)$ is closed under scalar multiplication. Now if $\mu, \nu \in V \cdot M_{b}(X)$, then $T(\mu)+T(\nu) \in C V_{0}(X)^{*}$, and hence there exists a $\tau \in V \cdot M_{b}(X)$ such that $T(\tau)$ $=T(\mu)+T(\nu)$. Taking $f \in C_{c}(X)$, we have

$$
\tau(f)=T(\tau)(f)=(T(\mu)+T(\nu))(f)=(\mu+\nu)(f)
$$

which implies $\tau=\mu+\nu$ and concludes the proof.

We now proceed to show that Theorem 3.1 does indeed generalize [13, Theorem 3.9]. To do this we utilize a topological space considered by Shirota in [12].

3.4. EXAMPLE. Denote the first uncountable ordinal by $\Omega$, the first infinite ordinal by $\omega$, and the space of all ordinals less than an ordinal $\alpha$ with the order topology by $W(\alpha)$. Define

$$
X=W(\Omega+1) \times W(\omega+1) \backslash A
$$

where $A=\{(\alpha, \omega): \alpha$ is a limit ordinal in $W(\Omega+1)\}$, and note that $X$ is locally compact and Hausdorff. Now let $\mathscr{P}$ denote the collection of all closed and relatively precompact (for a definition, see [12]) subsets of $X$, and for each $P \in \mathscr{P}$ let $\chi_{P}$ be the characteristic function of $P$. If $V=\left\{\lambda \chi_{P}: \lambda \geqq 0, P \in \mathscr{P}\right\}$, then $V$ is a Nachbin family on $X$ with $V \leqq C^{+}(X)$. Moreover, if there were a Nachbin family $U$ on $X$ with $U \subseteq C^{+}(X)$ and $U \approx V$, then $f \in U$ would imply that spt $(f)$ is relatively precompact. But $P=\{(\Omega, \alpha): \alpha \in W(\omega)\}$ is in $\mathscr{P}$ while if $f \in C^{+}(X)$ with $f(x) \geqq 1$ for $x \in P$, then spt $(f)$ is not relatively precompact.

It is the case, of course, that some restrictions are imposed on the class of Nächbin families under consideration in Theorem 3.1. One of these is the assumption that $C V_{0}(X)$ be Hausdorff which introduces in some sense a lower bound on this class, but will not exclude, for example, any $V$ for which $\omega_{V}$ is at least as fine as pointwise convergence. The other restriction is the hypothesis $V \leqq C^{+}(X)$, and the following example suggested by Professor N. Kimura shows this condition is not redundant.

3.5. EXAMPLE. If we let

$$
X=W(\Omega+1) \times W(\omega+1) \mid\{(\Omega, \omega)\} \quad \text { (the Tychonoff plank) }
$$

and define $v$ on $X$ by

$$
\begin{aligned}
v(\alpha, \beta) & =0, & & \alpha \neq \Omega, \\
& =\beta, & & \alpha=\Omega,
\end{aligned}
$$

then $v$ is a nonnegative u.s.c. function on $X$ which is unbounded, while $f \in C^{+}(X)$ implies $f$ is bounded.

We will conclude this section with a remark concerning $C V_{0}(X)^{*}$ where $V$ and $X$ are as in Theorem 3.1. As we have seen, in this case $C V_{0}(X)^{*} \subseteq M(X)$ and $\mu \in C V_{0}(X)^{*}$ implies there exist $v \in V, \nu \in M_{b}(X)$ so that $\mu=v \cdot \nu$ (a factorization 
of $\mu$ !). In general, this factorization is not unique as we show in the following example.

3.6. ExAmple. Let $X$ denote the reals with the usual topology, let $m$ denote Lebesgue measure on $X$, let $u(x)=\exp (|x|)$, and let $v(x)=\exp (2|x|)$. Now $u, v$, $1 / u, 1 / v \in C^{+}(X)$ and $\mu=(1 / u) \cdot m, \nu=(1 / v) \cdot m \in M_{b}(X)$ with $\mu \neq \nu$, but $m=u \cdot \mu=v \cdot \nu$.

4. Equicontinuous sets in $C V_{0}(X)^{*}$. In this section we will obtain a useful representation of a base for the equicontinuous subsets of $C V_{0}(X)^{*}$, namely $\left\{V_{v}^{0}: v \in V\right\}$; and then characterize the extremal points $\mathscr{E}\left(V_{v}^{0}\right)$ of the members of this base. It is not difficult to see that if $V$ is a Nachbin family on a locally compact space $X$ with $V \leqq C^{+}(X)$ and if $B=\left\{\mu \in M_{b}(X):\|\mu\| \leqq 1\right\}$, then $V_{v}^{0}$ is the weak-* closure in $C V_{0}(X)^{*}$ of $v \cdot B$. We will show that $v \cdot B$ is weak-* closed whence $V_{v}^{0}=v \cdot B$.

The following (probably known) lemma is included because of its role in the sequel.

4.1. LemMA. If $X$ is locally compact, if $u$ is a nonnegative u.s.c. function on $X$, and if there is an $f \in C^{+}(X)$ such that $u \leqq f$, then $u=\inf \left\{f \in C^{+}(X): f \geqq u\right\}$.

Proof. Fix $y \in X$, let $\varepsilon>0$, and choose $f \in C^{+}(X)$ with $f \geqq u$. We may assume $f(y)-u(y) \geqq \varepsilon$. Now $A=\{x \in X: u(x)<u(y)+\varepsilon / 4\}$ and $B=\{x \in X: f(x)>f(y)$ $-\varepsilon / 4\}$ are both open and $y \in A \cap B$. Take $\phi \in C_{c}(X)$ with $\phi(y)=1,0 \leqq \phi \leqq 1$, and spt $(\phi) \subseteq A \cap B$; define $\theta=(f(y)-u(y)-3 \varepsilon / 4) \phi$ and $g=f-\theta$. Thus $g \in C(X)$ and $g(y)-u(y)=3 \varepsilon / 4<\varepsilon$. Moreover, $x \in X \mid(A \cap B)$ implies $g(x)-u(x)=f(x)-u(x) \geqq 0$, while $x \in A \cap B$ implies $g(x)=f(x)-\theta(x)>f(y)-\varepsilon / 4-(f(y)-u(y)-3 \varepsilon / 4) \phi(x)$ $\geqq u(y)+\varepsilon / 2>u(x)$.

4.2. Lemma. If $X$ is locally compact, if $f, g \in C^{+}(X)$, and if for some $y \in X$ we have $f(y) g(y)<1$, then there exists $\phi \in C_{c}^{+}(X)$ satisfying $\phi(y)=g(y), \phi \leqq g$, and $\|f \phi\| \leqq 1$.

Proof. If $A=\{x \in X: f(x) g(x)<1\}$, then there exists $\theta \in C_{c}(X)$ so that $0 \leqq \theta \leqq 1$, $\theta(y)=1$, and spt $(\theta) \subseteq A$. It now follows that $\phi=g \theta$ is the desired function.

4.3. LEMMA. Let $X$ be locally compact, let $u$ be a nonnegative u.s.c. function on $X$ for which there is an $f \in C(X)$ such that $u \leqq f$, let $y \in X$, and let $\varepsilon>0$. If $g \in C^{+}(X)$ with $g u \leqq 1$, then there exists $\phi \in C_{c}^{+}(X)$ so that $\phi \leqq g, g(y)-\phi(y)<\varepsilon$, and $\|f \phi\| \leqq 1$ for some $f \in C(X)$ with $f \geqq u$.

Proof. Observe that we may assume $g(y) \neq 0$ and therefore can assume $\varepsilon<g(y)$. For $\eta=[2 g(y)(g(y)-\varepsilon)]^{-1} \varepsilon$, Lemma 4.1 yields an $f \in C^{+}(X)$ with $f \geqq u$ for which $f(y)-u(y)<\eta$. Now $g(y) f(y)-g(y) u(y)<\eta g(y)$ which implies $g(y) f(y)<1+\eta g(y)$. Define $\sigma=(\eta g(y)+1)^{-1} g$ and note that $\sigma(y) f(y)<1$. By Lemma 4.2, there exists $\phi \in C_{c}^{+}(X)$ with $\phi(y)=\sigma(y), \phi \leqq \sigma$, and $\|f \phi\| \leqq 1$. Since $\sigma \leqq g$, all that remains to be shown is that $g(y)-\phi(y)<\varepsilon$, but $g(y)-\phi(y)=(2 g(y)-\varepsilon)^{-1} g(y) \varepsilon<\varepsilon$. 
4.4. Lemma. Assume $X$ is locally compact and that $V$ is a Nachbin family on $X$ with $V \leqq C^{+}(X)$. For each $v \in V$ we have $V_{v}^{0} \subseteq v \cdot M_{b}(X)$, and $v \cdot \mu \in V_{v}^{0}$ if and only if $v \cdot|\mu| \in V_{v}^{0}$ whenever $\mu \in M_{b}(X)$.

Proof. It follows from Theorem 3.1 (and its proof) that $V_{v}^{0} \subseteq v \cdot M_{b}(X)$, while it is clear that $\mu \in M_{b}(X)$ and $v \cdot|\mu| \in V_{v}^{0}$ implies $v \cdot \mu \in V_{v}^{0}$. Now assume $v \cdot \mu \in V_{v}^{0}$ where $\mu \in M_{b}(X)$ and let $U=C^{+}(X)$. From a result in [13], $C U_{0}(X) \subseteq C V_{0}(X)$ and the identity map $i: C U_{0}(X) \rightarrow C V_{0}(X)$ is continuous. Choose $f \in U$ with $f \geqq v$ whence $i\left(V_{f}\right) \subseteq V_{v}$, and this implies $V_{v}^{0} \subseteq\left(i\left(V_{f}\right)\right)^{0}=i^{*^{-1}}\left(V_{f}^{0}\right)\left(i^{*}\right.$ is the transpose of $i)$; i.e., $i^{*}(v \cdot \mu) \in V_{f}^{0}$. It follows from [13, Lemma 3.8] that $V_{f}^{0}=f \cdot B$ where $B=\left\{\sigma \in M_{b}(X):\|\sigma\| \leqq 1\right\}$, and hence there is a $\sigma \in B$ so that $i^{*}(v \cdot \mu)=f \cdot \sigma$. But $\sigma \in B$ implies $|\sigma| \in B$, and this implies $f \cdot|\sigma| \in V_{f}^{0}$. Now there is a $\mu$-measurable function $h$ on $X$ with $|h|=1$ and $\mu=h \cdot|\mu|\left[11\right.$, p. 126], and hence $v \cdot \mu=i^{*}(v \cdot \mu)$ $=(v h) \cdot|\mu|=f \cdot \sigma=\left(f h^{\prime}\right) \cdot|\sigma|$ (where $h^{\prime}$ is the counterpart of $h$ for $\sigma$ ). Consequently,

$$
f \cdot|\sigma|=\left(v h \overline{h^{\prime}}\right) \cdot|\mu| \quad \text { which implies } v h \overline{h^{\prime}} \geqq 0 \text { a.e. }(|\mu|),
$$

and thus

$$
v=|v|=\left|v h \overline{h^{\prime}}\right|=v h \overline{h^{\prime}} \text { a.e. }(|\mu|) .
$$

Therefore $v \cdot|\mu|=f \cdot|\sigma|$ and we have that $v \cdot|\mu| \in V_{f}^{0}$ for every $f \in U$ with $f \geqq v$. If $g \in V_{v}$ with $g \geqq 0$, then for each $x \in X$ we have from Lemma 4.3 that $g(x)=$ $\sup \{\phi(x): \phi \in A\}$ where

$$
A=\left\{\phi \in C_{c}^{+}(X): \phi \leqq g, \phi \in V_{f} \text { for some } f \in U \text { with } f \geqq v\right\},
$$

and since $A$ is directed upward, $\int g v d|\mu|=\sup \left\{\int \phi v d|\mu|: \phi \in A\right\}[5$, p. 184]. But $\phi \in A$ implies $\int \phi v d|\mu| \leqq 1$ since $v \cdot|\mu| \in \bigcap\left\{V_{f}^{0}: f \in U, f \geqq v\right\}$, and hence $\int g v d|\mu| \leqq 1$. Clearly, this gives us that $v \cdot|\mu| \in V_{v}^{0}$ which completes the proof.

4.5. THEOREM. If $X$ is locally compact and if $V$ is a Nachbin family on $X$ with $V \leqq C^{+}(X)$, then $V_{v}^{0}=v \cdot B$ for each $v \in V$ where $B=\left\{\mu \in M_{b}(X):\|\mu\| \leqq 1\right\}$.

Proof. By Lemma $4.4, V_{v}^{0} \subseteq v \cdot M_{b}(X)$ and hence if $v \in V_{v}^{0}$, then there is a $\mu \in M_{b}(X)$ such that $\nu=v \cdot \mu$. Lemma 4.4 now gives us that $v \cdot|\mu| \in V_{v}^{0}$, and this fact together with Lemma 3.3 yield the conclusion of the theorem.

We now proceed to characterize the extremal points $\mathscr{E}\left(V_{v}^{0}\right)$ of the sets $V_{v}^{0}$ whenever $X$ and $V$ satisfy the hypotheses of Theorem 4.5. In the sequel, the point mass at $x \in X$ will be denoted by $\delta(x)$.

4.6. THEOREM. If $X$ is locally compact and if $V$ is a Nachbin family on $X$ with $V \leqq C^{+}(X)$, then

$$
\mathscr{E}\left(V_{v}^{0}\right)=\{\lambda v(x) \delta(x): x \in N(v),|\lambda|=1\}
$$

for each $v \in V$.

Proof. Fix $v \in V$, let $E=\{\lambda v(x) \delta(x): x \in N(v),|\lambda|=1\}$, and note that $E \subseteq V_{v}^{0}$. We first show that $E \subseteq \mathscr{E}\left(V_{v}^{0}\right)$, and to this end choose $\mu=\lambda v(x) \delta(x) \in E$ and suppose 
$\mu=(1 / 2)(\sigma+\tau)$ where $\sigma, \tau \in V_{v}^{0}$. Now $\sigma=v \cdot \alpha, \tau=v \cdot \beta$ where $\alpha, \beta \in M_{b}(X)$ with $\|\alpha\|,\|\beta\| \leqq 1$ by Theorem 4.5 , and without loss of generality we may assume $|\alpha|(X \backslash N(v))=|\beta|(X \backslash N(v))=0$. Thus $\lambda v(x) \delta(x)=((1 / 2) v) \cdot(\alpha+\beta)$, and since it will suffice to assume $\lambda=1$, we claim that in this case $\delta(x)=(1 / 2)(\alpha+\beta)$. To see this, let $A$ be open with $x \notin A$ and choose $\phi \in C_{c}^{+}(X)$ with spt $(\phi) \subseteq A$. Then

$$
\begin{aligned}
\int \phi d(\alpha+\beta) & =\int_{N(v)} \phi d(\alpha+\beta)=\int_{N(v)}(\phi / v) d(\sigma+\tau) \\
& =2 v(x) \int_{N(v)}(\phi / v) d \delta(x)=2 \phi(x)=0,
\end{aligned}
$$

which implies $|\alpha+\beta|(A)=0[5$, p. 202]. Hence spt $(\alpha+\beta)=\{x\}$, while $(\alpha+\beta)(\{x\})$ $=v(x)^{-1}[v(x)(\alpha+\beta)(\{x\})]=2 \delta(x)$. Since $\delta(x)$ is an extremal point of the unit ball of $M_{b}(X), \delta(x)=\alpha=\beta$ from whence $v \cdot \delta(x)=v \cdot \alpha=v \cdot \beta$, and this implies $\mu \in \mathscr{E}\left(V_{v}^{0}\right)$.

We used above a characterization of the extremal points of the unit ball in $M_{b}(X)$ [3] which is an extension of the well-known Arens-Kelley result. To complete the proof, we adapt the argument which Conway [3] used to obtain this extension. Now take $\mu \in \mathscr{E}\left(V_{v}^{0}\right)$ and, using Theorem 4.5 , write $\mu=v \cdot \nu$ where $\nu \in M_{b}(X)$ with $\|\nu\| \leqq 1$ and $|\nu|(X \mid N(v))=0$. Note that in this case $\|\nu\|=1$. Consider $\mathscr{K}=$ $\{K \subseteq X: K$ is compact, $|\nu|(K)>0\}$ and observe that $\mathscr{K}$ is not void. If $K_{1}, K_{2} \in \mathscr{K}$ and if $K_{3}=X \mid\left(K_{1} \cup K_{2}\right)$, then $\mu=\sum_{i=1}^{3}|\nu|\left(K_{i}\right) v \cdot \mu_{i}$ where

$$
\begin{aligned}
\mu_{i}(A) & =|\nu|\left(K_{i}\right)^{-1} \nu\left(A \cap K_{i}\right), & & |\nu|\left(K_{i}\right) \neq 0, \\
& =0, & & |\nu|\left(K_{i}\right)=0,
\end{aligned} \quad i=1,2,3,
$$

for Borel sets $A$. Hence $\mu=v \cdot \mu_{1}$ which implies $|\nu|\left(K_{2}\right)=\left|\mu_{1}\right|\left(K_{2} \cap N(v)\right)=$ $|\nu|\left(K_{1}\right)^{-1}|\nu|\left(K_{1} \cap K_{2}\right)$ from whence $K_{1} \cap K_{2}$ is not void. If $K \subseteq K_{1} \mid K_{2}$ is compact, then $|\nu|(K)=0$, and this implies $|\nu|\left(K_{1} \mid K_{2}\right)=0$; therefore $|\nu|\left(K_{1} \cap K_{2}\right)=|\nu|\left(K_{1}\right)>0$, and thus $K_{1} \cap K_{2} \in \mathscr{K}$. It easily follows that $\mathscr{K}$ is closed under finite intersections and hence there is an $x \in \bigcap\{K: K \in \mathscr{K}\}$. If $A$ is open and $x \notin A$ and if $\phi \in C_{c}^{+}(X)$ with spt $(\phi) \subseteq A$, then $\left|\int \phi d \mu\right| \leqq\|\phi v\||\nu|(\operatorname{spt}(\phi))=0$, and from this we have that spt $(\mu)=\{x\}$. Now $\mu \neq 0$ implies $x \in N(v)$, and so $\mu=\alpha \delta(x)$ where $\alpha=v(x) \nu(X)$, and $|\nu(X)|=1$ since $\|\nu\|=1$. Consequently, $\mu \in E$ and the proof is complete.

5. Applications and observations. One of the more immediate applications of the preceding development is the following generalization (see Example 3.4) of the author's representation theorem for tensor products of weighted spaces [13]. The proof will be omitted since it is exactly as in [13] with the exception of using Theorem 4.6 in place of the corresponding result in [13].

5.1. THEOREM. Let $X$ and $Y$ be locally compact. If $U$ is a Nachbin family on $X$ with $C_{c}^{+}(X) \leqq U \leqq C^{+}(X)$ and if $V$ is a Nachbin family on $Y$ with $C_{c}^{+}(Y) \leqq V \leqq C+(Y)$, then the biequicontinuous completed tensor product $C U_{0}(X) \tilde{\otimes} C V_{0}(Y)$ is topologically isomorphic to $C W_{0}(X \times Y)$ where $W=\{u \times v: u \in U, v \in V\}$ (here $u \times v$ is the function on $X \times Y$ defined by $u \times v(x, y)=u(x) v(y))$. 
We now consider a class of Nachbin families $V$ on a locally compact space $X$ which have the property that $V \cdot M_{b}(X)=M_{b}(X)$. In particular, if $V \subseteq B^{+}(X)$, then surely $V \cdot M_{b}(X) \subseteq M_{b}(X)$, while the fact that $M_{b}(X)=C_{0}^{+}(X) \cdot M_{b}(X)[1$, p. 99] implies every $\mu \in M_{b}(X)$ defines a continuous linear functional on $C V_{0}(X)$ whenever $C_{0}^{+}(X) \leqq V$ in view of [13, Theorem 3.1]. An application of Theorem 3.1 now yields the following result.

5.2. THEOREM. If $X$ is locally compact and if $V$ is a Nachbin family on $X$ with $C_{0}^{+}(X) \leqq V \subseteq B^{+}(X)$, then $C V_{0}(X)^{*}=V \cdot M_{b}(X)=M_{b}(X)$.

This result leads us to the question posed below. Buck [1] asked if $\left(C_{b}(X), \beta\right)$ is a Mackey space, and Conway [4] gave an affirmative answer in case $X$ is paracompact. So if $X$ is locally compact and paracompact, then $\tau\left(C_{b}(X), M_{b}(X)\right)$ is the weighted topology $\beta$ determined by the Nachbin family $C_{0}^{+}(X)$. This raises the question of when $\tau\left(C_{b}(X), M_{b}(X)\right)$ is a weighted topology determined by a Nachbin family $V$ on $X$ for which $C V_{0}(X)=C_{b}(X)$. The answer to this question (see Theorem 5.5) is an immediate consequence of the following two lemmas.

5.3. Lemma. If $X$ is locally compact and if $V$ is the set of all nonnegative u.s.c. functions on $X$ which vanish at infinity, then $V$ is a Nachbin family on $X$ and $V \approx C_{0}^{+}(X)$.

We remark before beginning the proof that it is of some interest to consider this result in light of Example 3.5.

Proof. It is, of course, clear that $V$ is a Nachbin family on $X$ and that $C_{0}^{+}(X) \subseteq V$. If $v \in V$, then $v \in B(X)$, and so to complete the proof it will suffice to assume $\|v\|=1$ and show there is a $\phi \in C_{0}^{+}(X)$ such that $v \leqq \phi$. Now $K_{n}=\left\{x \in X: v(x) \geqq 2^{-n}\right\}$ is compact for each $n=1,2, \ldots$, and hence for each $n$ there is a $\phi_{n} \in C_{c}(X)$ such that $0 \leqq \phi_{n} \leqq 1$ and $\phi_{n}\left(K_{n}\right)=1$. If we define $\phi=\sum_{n=1}^{\infty} 2^{-(n-1)} \phi_{n}$, then $\phi \in C_{0}^{+}(X)$. Moreover, if $x \in N(v)$, then there exists an $n$ such that $x \in K_{n}$ and $v(x) \leqq 2^{-(n-1)}$, while in this case $\phi(x) \geqq 2^{-(n-1)}$.

5.4. Lemma. Let $X$ be locally compact and let $V$ be a Nachbin family on $X$. If $C V_{0}(X)=C_{b}(X)$ and if $\omega_{V}$ is at least as fine as $\beta$, then $V \approx C_{0}^{+}(X)$.

Proof. It follows from [13, Theorem 3.3] that $C_{0}^{+}(X) \leqq V$, while $V \leqq C_{0}^{+}(X)$ follows from Lemma 5.3 together with the fact that $1 \in C V_{0}(X)$.

5.5. THEOREM. If $X$ is locally compact, then the following are equivalent:

(1) $\left(C_{b}(X), \beta\right)$ is a Mackey space.

(2) There exists a Nachbin family $V$ on $X$ such that

$$
C V_{0}(X)=\left(C_{b}(X), \tau\left(C_{b}(X), M_{b}(X)\right)\right) .
$$

Several other questions remain unanswered, however. For example, one might ask which pairs $(V, X)$ where $V$ is a Nachbin family on $X$ imply $C V_{0}(X)$ is a Mackey space, a barrelled space, etc. 
Theorem 5.2 gave a class of Nachbin families $V$ on a locally compact space $X$ for which $C V_{0}(X)^{*}=M_{b}(X)$. We also note the following easily proved result.

5.6. THEOREM. If $X$ is locally compact and if $V$ is a Nachbin family on $X$ with $C_{c}^{+}(X) \leqq V \subseteq B^{+}(X)$, then $M_{c}(X) \subseteq V \cdot M_{b}(X)=C V_{0}(X)^{*} \subseteq M_{b}(X)$ where $M_{c}(X)$ is the space of Radon measures with compact support. Moreover, if $V \approx C_{c}^{+}(X)$, then $C V_{0}(X)^{*}=M_{c}(X)$.

Finally, consider $C(X)$ with the compact-open (co-op) topology and define $\delta: X \rightarrow(C(X), \text { co-op })^{*}=M_{c}(X)$ by $\delta(x)(f)=f(x)$. It is well known that $\delta$ is a homeomorphism onto its image when the range has its weak-* topology (e.g., see [16, p. 266]). Varadarajan [15] has obtained other results for the map $\delta$ in a somewhat different setting. In particular, he has shown that if $X$ is a metric space, then $\delta(X)$ is sequentially closed in $\left(\left(C_{b}(X),\|\cdot\|\right)^{*}, \sigma\left(\left(C_{b}(X),\|\cdot\|\right)^{*}, C_{b}(X)\right)\right)[15$, p. 197]. With only the assumption that $X$ be locally compact and Hausdorff, we obtain the following stronger result.

5.7. THEOREM. Let $X$ be locally compact and let $V$ be a Nachbin family on $X$ with $C_{c}^{+}(X) \leqq V \leqq C_{0}^{+}(X)$. If $\delta: X \rightarrow M_{b}(X)$ is defined by $\delta(x)$ equals the point mass at $x$ for each $x \in X$, then $\delta$ is a one-to-one continuous closed map into $\left(C V_{0}(X)^{*}, \sigma\left(C V_{0}(X)^{*}, C V_{0}(X)\right)\right)$.

Proof. That $\delta(X) \subseteq C V_{0}(X)^{*}$ follows from Theorem 5.6, while it is clear that $\delta$ is one-to-one and continuous. Let $F$ be a closed subset of $X$, and observe that it will suffice to show $\delta(F)$ is $\sigma\left(M_{b}(X), C_{b}(X)\right)$-closed since $C V_{0}(X)^{*} \subseteq M_{b}(X)$ by Theorem 5.6 while $C_{b}(X) \subseteq C V_{0}(X)$. To this end, let $\mu$ be a $\sigma\left(M_{b}(X), C_{b}(X)\right)$ limit point of $\delta(F)$ in $M_{b}(X)$, and note that spt $(\mu)$ is not void since $1 \in C_{b}(X)$ implies $\mu \neq 0$. Choose $y \in \operatorname{spt}(\mu)$ and a net $\left\{\delta\left(x_{i}\right)\right\}$ in $\delta(F)$ with $\delta\left(x_{i}\right) \rightarrow \mu$, and suppose the net $\left\{x_{i}\right\}$ does not converge to $y$. In this case there is an open neighborhood $A$ of $y$ for which $\left\{x_{i}\right\}$ is not eventually in $A$, and there is a $\phi \in C_{c}^{+}(X)$ such that spt $(\phi) \subseteq A$ with $\left|\int \phi d \mu\right|>0\left[5\right.$, p. 202]. Now let $\varepsilon>0$, choose $i_{0}$ so that $i \geqq i_{0}$ implies $\left|\int \phi d \mu-\phi\left(x_{i}\right)\right|<\varepsilon$, and choose $j \geqq i_{0}$ such that $x_{j} \notin A$. Thus

$$
\left|\int \phi d \mu\right| \leqq\left|\int \phi d \mu-\phi\left(x_{j}\right)\right|+\phi\left(x_{j}\right)<\varepsilon
$$

which contradicts the choice of $\phi$. Hence $\mu=\delta(y)$ and the proof is complete.

\section{REFERENCES}

1. R. C. Buck, Bounded continuous functions on a locally compact space, Michigan Math. J. 5 (1958), 95-104. MR 21 \#4350.

2. H. S. Collins and J. R. Dorroh, Remarks on certain function spaces, Math. Ann. 176 (1968), 157-168. MR 36 \#5694.

3. J. B. Conway, The strict topology and compactness in the space of measures, Ph.D. Dissertation, Louisiana State University, Baton Rouge, 1965.

4. - The strict topology and compactness in the space of measures. II, Trans. Amer. Math. Soc. 126 (1967), 474-486. MR 34 \#6503. 
5. R. E. Edwards, Functional analysis theory and applications, Holt, Rinehart and Winston, New York, 1965. MR 36 \#4308.

6. L. Gillman and M. Jerison, Rings of continuous functions, The University Series in Higher Math., Van Nostrand, Princeton, N. J., 1960. MR 22 \#6994.

7. L. Nachbin, Weighted approximation for algebras and modules of continuous functions: real and self-adjoint complex cases, Ann. of Math. (2) 81 (1965), 289-302. MR 31 \#628.

8. - Elements of approximation theory, Math. Studies, no. 14, Van Nostrand, Princeton, N. J., 1967. MR 36 \#572.

9. J. B. Prolla, Weighted approximation and operator algebras, Ph.D. Dissertation, New York University, 1968.

10. A. P. Robertson and W. J. Robertson, Topological vector spaces, Cambridge Tracts in Math. and Math. Phys., no. 53, Cambridge Univ. Press, New York, 1964. MR 28 \#5318.

11. W. Rudin, Real and complex analysis, McGraw-Hill, New York, 1966. MR 35 \#1420.

12. T. Shirota, On locally convex vector spaces of continuous functions, Proc. Japan Acad. 30 (1954), 294-298. MR 16 \#275.

13. W. H. Summers, A representation theorem for biequicontinuous completed tensor products of weighted spaces, Trans. Amer. Math. Soc. 146 (1969), 121-131.

14. B. A. Taylor, Some locally convex spaces of entire functions, Proc. Sympos. Pure Math., vol. 11, Amer. Math. Soc., Providence, R. I., 1968, pp. 431-467.

15. V. S. Varadarajan, Measures on topological spaces, Mat. Sb. 55 (97) (1961), 35-100; English transl. Amer. Math. Soc. Transl. (2) 48 (1965), 161-228. MR 26 \#6342.

16. S. Warner, The topology of compact convergence on continuous function spaces, Duke Math. J. 25 (1958), 265-282. MR 21 \#1521.

\section{Louisiana State University, \\ Baton Rouge, Louisiana 70803 \\ UNIVERSITY OF ARKansas, \\ FAYETTEVILLE, Arkansas 72701}

